\title{
The role of punch biopsy in the management of carcinoma of the cervix in a low resource centre
}

\author{
Adekunle Olanrewaju Oguntayo \\ Department of Gynecology \& Obstetrics, ABU Teaching Hospital, Shika, Zaria \\ Email: email-fayokunmi@yahoo.co.uk
}

Received 3 September 2013; revised 3 October 2013; accepted 11 October 2013

Copyright $\odot 2013$ Adekunle Olanrewaju Oguntayo. This is an open access article distributed under the Creative Commons Attribution License, which permits unrestricted use, distribution, and reproduction in any medium, provided the original work is properly cited.

\begin{abstract}
Background: Carcinoma of the cervix is the commonest malignant tumor of the female genital tract in Northern Nigeria and the leading cause of cancerrelated death in women in developing countries. The predicament is further worsened by poor uptake of cytological screening programs, which are either not available or where available are underutilized due to lack of widespread awareness and the cost as an average Nigerian earns less than a dollar per day. In addition to the above constrains, majority of these patients also presents late to the hospital for intervention. Objective: To emphasize the significance of punch biopsy and clinical staging of advanced carcinoma of the cervix and see how we can shift from our traditional, Examination under Anesthesia (EUA) to Clinical staging and punch biopsy for advanced carcinoma of the cervix. Methodology: Review of existing literature and experience from our own center. Results: The punch biopsy has $88.8 \%$ positive predictive value in making a diagnosis of cancer of the cervix. Late presentation was as high as 81.7 percent for carcinoma of the cervix in our centre. The patients present late for care; majority actually presents in stages III \& IV when little or nothing can be done for them. Conclusion: In view of these predicaments that face a gynecological oncologist practicing in low resources settings like ours, there is a need to consider the role of punch biopsy, clinical staging and radiological assessment for making a diagnosis, especially among those women who cannot afford EUA or are having renal complication that makes EUA unachievable.
\end{abstract}

Keywords: Punch; Biopsy; Carcinoma; Cervix \& Low Resource

\section{THE ROLE OF PUNCH BIOPSY IN THE MANAGEMENT OF CARCINOMA OF THE CERVIX}

Carcinoma of the cervix is the commonest malignant tumor of the female genital tract in Northern Nigeria and the leading cause of cancer-related death in women in developing countries [1].

Worldwide, cervical carcinoma is the second commonest female malignancy [2]. However cancer of the cervix uteri is the most frequent neoplasm among women in developing nations [1]. Over 400,000 new cases are diagnosed annually with a mortality of 230,000 from developing nations [2].

This disease entity is said to be more common in women of low socioeconomic group, who are equally uneducated [3]. This is not unconnected with the low literacy level of the women in the country generally [3].

The predicament is further worsened by poor uptake of cytological screening programs [3], which are either not available or where available are underutilized due to lack of widespread awareness. The cost also makes the screening out of reach of an average individual who earns less than a dollar per day [4].

In addition to the above constrains, majority of these patients also presents late to the hospital for intervention. Their late presentation could either be faith based (as in accepting whatever befalls them, as the act of God) or the use of traditional medication as alternative treatment $[5,6]$.

Late presentation was as high as 81.7 percent for carcinoma of the cervix in our centre [3,7]. The patients present late for care; majority actually presents in stages III \& IV when little or nothing can be done for them $[5,6]$.

At the time of presentation majority of them have developed severe anemia from on and off vagina bleeding, deranged electrolyte from obstructive uropathy, while oth- 
ers presents with features of metastasis [6].

A cervical biopsy is a procedure performed to obtain tissue from the cervix for pathological analysis towards making a definitive diagnosis of a cervical pathology. This procedure can be done using various approaches. A punch biopsy is an office procedure usually carried out along with colposcopy in the management of pre-invasive carcinoma of the cervix uteri. Other forms of biopsy are usually done under general anesthesia such as cone or wedge biopsy [7].

The punch biopsy has $88.8 \%$ positive predictive value in making a diagnosis of cancer of the cervix [3]. The inadequate samplings were usually as a result of either of the following; Obscuring hemorrhage to extensive tissue necrosis, non representative tissue biopsy and poor tissue preparation and transportation [3].

The goal standard practice is examination under anaesthesia (EUA) staging and biopsy. This is not usually visible in patients in our settings, because majority of them at presentation are not fit for surgery.

In addition, the procedure is more expensive than the treatment in many centers, i.e. Radiotharapy/Chemoradiation therapy course. The unfortunate or sad part is that many of them having been optimized (blood transfusion) for EUA will still require more blood before treatment could be commenced. Generally our patients receive 2 7 units of blood in the course of their management with an average of four units [7]. Blood is very difficult to come by in this era of HIV/AIDS. We also need to consider other side effect of blood transfusion.

In some instances our patients die only at the point of making diagnosis, either for lack of funds to continue treatment or lack of blood to resuscitate them, while some others die from renal complication (Obstructive
Uropathy).

In view of this predicament that faces a gynecological oncologist practicing in low resources settings like ours, there is a need to consider the role of punch biopsy, clinical staging and radiological assessment for making a diagnosis, especially among those women who cannot afford EUA or are having renal complication that makes EUA unachievable.

This option would actually offer these women an opportunity for treatment early enough and might go a long way to improve the quality of life in these women.

\section{REFERENCES}

[1] Mohammed, A., Ahmed, S.A., Oluwole, O.P. and Avidime, S. (2006) Malignant tumours of the female genital tract in Zaria, Nigeria: Analysis of 513 cases. Annals of African Medicine, 5, 93-96.

[2] Canavan, T. and Doshi, N.R. (2000) Cervical cancer. American Family Physician, 1, 1369-1382.

[3] Samaila, M.O.A., Adesiyun, A.G. and Kolawole, A.O. (2007) Evaluation of cervical punch biopsy in the diagnosis of cervical cancer. Highland Medical Research, 5, 33-37.

[4] The Millennium Development Goals Report of 2007.

[5] Adewuyi, S.A., Shittu, S.O. and Rafindadi, A.H. (2007) Cervical canaer in HIV seropositive patients. West Africa Journal of Radiology, 14, 31-38.

[6] Obafunwa, J.O., Sagay, A.S. and Otubu, J.A.M. (1991) Prevalence of cervical intraepithelial neoplasia. Tropical Journal of $O \& G, 9,16-17$.

[7] Oguntayo, O.A, Zayyan, M., Kolawole, A.O.D., Adewuyi, S.A., Ismail, H. and Koledade, K. (2011) Cancer of the cervix in Zaria, Northern Nigeria. Ecancer, 5, 219. 